\title{
Monitoramento Inteligente de Consumo Energético em Ambiente Residencial utilizando IoT
}

\author{
Valdenir S. Severino ${ }^{1}$, Danilo R. Vasconcelos ${ }^{2}$, Rossana M. C. Andrade $^{1 *}$ \\ ${ }^{1}$ Grupo de Redes, Engenharia de Software e Sistemas (GREaT) \\ Mestrado e Doutorado em Ciência da Computação (MDCC), \\ Universidade Federal do Ceará (UFC) - Fortaleza, Ceará, Brasil \\ ${ }^{2}$ Instituto Federal de Educação, Ciência e Tecnologia do Ceará (IFCE) \\ - Morada Nova, Ceará, Brasil \\ \{valdenirseverino, rossana\}egreat.ufc.br \\ danilo.reiseifce.edu.br
}

\begin{abstract}
Energy waste in Brazil has been growing in recent years and one reason for this increase is the fact that the end-user is usually unaware of the amount of energy consumed by their home appliances. On the other hand, the Internet of Things (IoT) brings concepts, such as smart objects with their data collection and analysis, which can help end-users in the identification of their home appliances' energy consumption. Therefore, this work proposes an intelligent monitoring system capable of discovering the devices connected to the power grid in real-time. Using a smart device, installed in the power grid, our proposed system extracts the consumption characteristics of the appliances and send them to a classification algorithm in the cloud. We performed controlled experiments to evaluate the proposed solution and, as a result, all appliances connected to the power grid were identified, allowing the end-users to monitor their home energy consumption.
\end{abstract}

Resumo. O desperdício de energia elétrica no Brasil vem crescendo nos últimos anos e um dos motivos para este aumento é o fato de que o consumidor final normalmente não tem conhecimento sobre a quantidade de energia consumida por seus eletrodomésticos. Por outro lado, a Internet das Coisas (IoT) traz conceitos, como objetos inteligentes, com sua coleta e análise de dados, que podem ajudar usuários finais na identificação do consumo de energia de seus eletrodomésticos. Portanto, este trabalho propõe um sistema de monitoramento inteligente capaz de descobrir os eletrodomésticos conectados à rede elétrica em tempo real. Através de um dispositivo inteligente, instalado na rede elétrica, esse sistema extrai as características de consumo dos eletrodomésticos e as envia para um algoritmo de classificação na nuvem. Nós realizamos experimentos controlados para avaliar a solução proposta e, como resultado, todos os aparelhos conectados à rede elétrica foram identificados, permitindo que os consumidores monitorem os gastos de energia da sua casa.

*Bolsista do CNPq de Produtividade em Desenvolvimento Tecnológico e Extensão Inovadora (DT) 2 


\section{Introdução}

A eficiência energética está se tornando um problema desafiador devido às crescentes demandas [Ma et al. 2017] e o desperdício de energia gera prejuízo de cerca de 71 milhões de reais por dia [Abesco 2017]. Dessa forma, torna-se cada vez mais necessário a utilização e desenvolvimento de estratégias voltadas para o monitoramento do uso de energia elétrica, permitindo que o usuário possa acompanhar o consumo de cada eletrodoméstico em tempo real.

Uma maneira eficiente de gestão energética residencial pode ser obtida através da identificação e monitoramento do consumo de energia elétrica dos eletrodomésticos presentes nas residências [Ma et al. 2017], o que permite ao usuário a verificação da quantidade de energia consumida pelos eletrodomésticos e seu correto funcionamento, bem como desperdício de energia, monitoramento esse, realizado por meio da Internet das Coisas (do inglês, Internet of Things ou IoT), que por sua vez define-se como uma interconexão entre dispositivos ou objetos inteligentes conectados a sensores e atuadores, provendo a habilidade de compartilhar informações na Internet [Gubbi et al. 2013].

É possível perceber a necessidade da adoção de sensores conectados ou acoplados aos eletrodomésticos, entretanto, observa-se limitações na viabilidade devido ao custo de tais equipamentos. Alternativamente, adotar um único sensor ou dispositivo inteligente que colete informações de consumo de toda a residência pode ser mais econômica, contudo o desafio está na complexidade agregada advinda do funcionamento simultâneo de vários eletrodomésticos [Zhuang et al. 2019], o que exige criteriosidade de análise no sentido de desagregar o sinal de consumo.

O que se propõe neste trabalho é o monitoramento inteligente do consumo energético residencial, adotando uma estratégia que consiste na utilização de um algoritmo de identificação de eletrodomésticos por meio da análise das componentes harmônicas presentes no sinal da corrente elétrica, obtidas a partir de um único dispositivo inteligente de coleta e processamento de dados instalado na rede elétrica residencial. Por meio deste dispositivo inteligente é realizado o monitoramento de toda a rede elétrica residencial, permitindo a descoberta de quais eletrodomésticos estão ligados, mesmo que estes não possuam nenhum tipo de inteligência. Além disso, as informações coletadas são disponibilizadas através de uma aplicação móvel, permitindo assim um maior controle por parte do usuário.

Este artigo está organizado da seguinte forma: a fundamentação teórica é apresentada na Seção 2; os trabalhos relacionados são apresentados na Seção 3; os módulos que compõem o sistema de monitoramento elétrico residencial são descritos na Seção 4 ; os experimentos e os resultados obtidos com o algoritmo de identificação de eletrodomésticos são descritos na Seção 5; e, por fim, na Seção 6, a conclusão e os trabalhos futuros são apresentados.

\section{Referencial Teórico}

Nesta seção são introduzidos conceitos relevantes para este trabalho, como objetos inteligentes e as componentes harmônicas de sinais elétricos. 


\subsection{Objetos Inteligentes}

A Internet das Coisas é um paradigma que consiste em conectar uma variedade de objetos na Internet, permitindo a interação e cooperação entre eles, com a finalidade de atingir objetivos comuns [Atzori et al. 2010]. Segundo Bandyopadhyay et al. (2011), os objetos ou "coisas" são dispositivos, físicos ou virtuais, que possuem identidades, atributos e personalidades virtuais, capazes de utilizar interfaces inteligentes, realizam o processamento de dados através de um microcontrolador e possuem um conversor analógico-digital para receber os sinais obtidos pelos sensores [Santos et al. 2016].

Em uma residência inteligente, por exemplo, o usuário pode monitorar e controlar seus eletrodomésticos através da Internet. Os mesmos atuam como objetos inteligentes, integrando uma rede de troca de informações entres eles e a Internet Gaikwad et al. 2015]. No entanto, nem sempre os eletrodomésticos possuem alguma tecnologia disponível que os classifiquem como objetos inteligentes, sendo necessária a utilização de dispositivos externos, tais como tomadas inteligentes [Jacobs e Maun 2019], que extraiam as informações úteis destes eletrodomésticos e publiquem-nas na Internet.

\subsection{Componentes Harmônicas do Sinal Elétrico}

A forma de onda da tensão e corrente elétrica consumida pelos usuários residenciais, em condições ideais, deveria ser fornecida como uma tensão puramente senoidal, com frequência e amplitude constantes. Entretanto, na prática, este sinal pode apresentar deformações causadas por diversos componentes elétricos, com características nãolineares, comumente conectados na rede elétrica. Os eletrodomésticos construídos com componentes eletrônicos (transistores, tiristores, diodos, etc) não aproveitam a energia em seu formato senoidal de onda, modificando o sinal da rede e isso ocorre porque estes componentes apresentam uma relação não linear entre a corrente e a tensão, originando componentes de frequência conhecidas como harmônicas [Azevedo et al. 2019].

As componentes harmônicas presentes no sinal de corrente podem ser extraídas através da Transformada Rápida de Fourier (do inglês Fast Fourier Transform, ou FFT). A FFT permite que qualquer sinal possa ser decomposto em uma componente de mesma frequência que a do sinal original, denominada onda fundamental e em outras componentes de frequências múltiplas da fundamental (componentes harmônicas). A presença ou não destas componentes de frequência e o valor sua amplitude podem indicar características únicas do tipo de eletrodoméstico conectado na rede elétrica [Temneanu 2014].

\section{Trabalhos Relacionados}

Esta seção apresenta os trabalhos relacionados que abordam o monitoramento e identificação de eletrodomésticos conectados à rede elétrica, focos da proposta deste artigo.

[Semwal et al. 2014] analisam o sinal das harmônicas presentes no sinal de corrente demandado pelo acionamento de determinados eletrodomésticos. A extração do sinal de consumo é feita através da leitura de um sensor de corrente não invasivo conectado em um único ponto no circuito elétrico de teste. O sinal de corrente coletado é processado através da Transformada Rápida de Fourier, obtendo assim o sinal das harmônicas, que, por sua vez, é analisado por 3 tipos diferentes de classificadores: Naive Bayes, SVM e Rede Neural. A solução apresentada por [Semwal et al. 2014] é mais complexa, em 
comparação a solução descrita neste artigo, no que se refere ao treinamento do algoritmo classificador, no qual demanda a coleta de um número maior de amostras de consumo de eletrodomésticos ligados ao circuito elétrico, consequentemente mais combinações envolvendo sinais de consumo.

A próxima seção descreve a utilização do monitoramento inteligente na tentativa de solucionar o problema de [Semwal et al. 2014] realizando pré-processamento do sinal de consumo e análise das variações das componentes harmônicas à medida que os eletrodomésticos são ligados ou desligados da rede elétrica, dessa forma o algoritmo de classificação necessita conhecer apenas o sinal específico de cada equipamento analisado.

Já no trabalho de [Ma et al. 2017] é desenvolvido um sistema de detecção de eletrodomésticos através de algoritmos de mineração de dados e aprendizado de máquina. O trabalho apresenta o conceito de impressão digital (do inglês, fingerprint) do eletrodoméstico. Esta impressão digital pode ser de dois tipos: o primeiro, baseado em equipamento, retrata as características de comportamento exclusivas do aparelho, tais como potência consumida e fator de potência. $\mathrm{O}$ segundo, baseado em contexto, reflete o padrão de uso dos eletrodomésticos, como hora, dia da semana, clima e temperatura. Essas medidas de contexto podem indicar os períodos de tempo de utilização dos eletrodomésticos, por exemplo, muitos utensílios de cozinha são frequentemente utilizados de manhã e à noite durante a semana.

Assim como no trabalho de [Ma et al. 2017], [Jacobs e Maun 2019] realiza as medições do consumo através de sensores de corrente e tensão, instalados em tomadas inteligentes. A diferença entre os dois é que o segundo trabalho apresenta um método para a identificação de eletrodomésticos específicos, exemplificado por uma máquina de lavar roupa, dentre o conjunto de outros eletrodomésticos presentes em uma residência. Segundo os autores, a maior parte da energia consumida em uma casa (aproximadamente $80 \%$ ) é composta por apenas alguns aparelhos, sendo o eletrodoméstico identificado através de um algoritmo de clusterização, no qual são analisados os valores de potência ativa e reativa.

A vantagem de abordagens que utilizam as tomadas inteligentes, como as duas últimas citadas anteriormente é a precisão relacionada à extração das medidas elétricas de consumo dos eletrodomésticos, uma vez que cada tomada pode se relacionar a um equipamento de forma individual, facilitando desta forma a identificação dos que estão funcionando em tempo real. A desvantagem está na necessidade de modificações no circuito elétrico residencial, resultando em um alto custo de implantação. Uma abordagem mais econômica de identificação de eletrodomésticos é através da utilização de um único sensor para monitorar toda a rede elétrica residencial, conforme é feito na nossa solução apresentada na próxima seção.

\section{Monitoramento Inteligente de Consumo Elétrico Residencial}

Neste trabalho é proposto um sistema de monitoramento inteligente de consumo energético em ambiente residencial, indoor, usando conceitos de IoT. Este sistema está dividido em três componentes: o primeiro trata-se de um dispositivo inteligente de coleta, processamento e envio das características de consumo dos eletrodomésticos; o segundo, um serviço em nuvem responsável por executar o algoritmo de identificação de eletrodomésticos, além de armazenar as informações referentes ao perfil de consumo energético 
da casa; e, por fim, o terceiro, uma aplicação móvel, que serve de dashboard, permitindo assim a visualização das informações de consumo energético residencial. A Figura 1 apresenta uma visão geral da solução proposta.

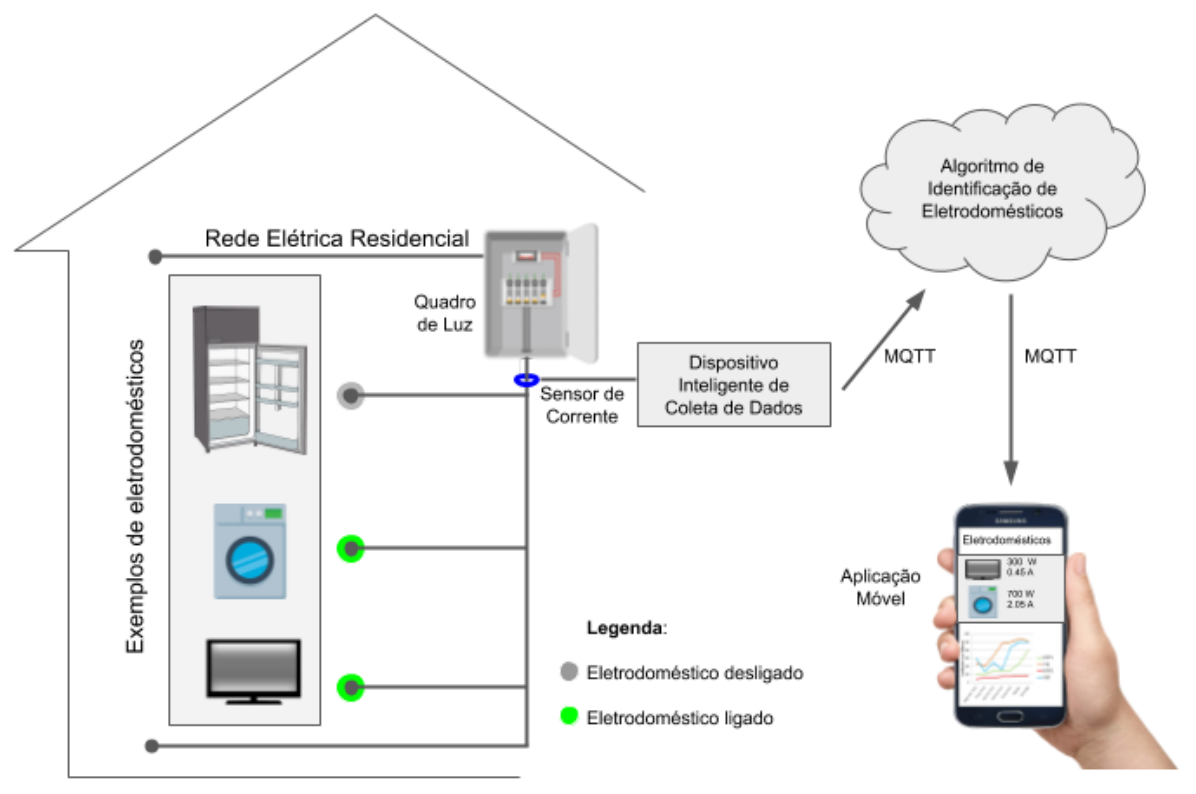

Figura 1. Visão Geral da Solução Proposta

Os protocolos de comunicação são responsáveis por facilitar a comunicação dos objetos em uma rede IoT [Andrade et al. 2017]. Na solução proposta deste artigo, a troca de informações é realizada através do protocolo MQTT. Este protocolo é bastante utilizado no contexto de IoT devido à sua facilidade de implementação, robustez e eficiência [Yokotani e Sasaki 2017]. O protocolo MQTT segue o modelo publish/subscriber e a troca de mensagens é realizada através de estruturas conhecidas como tópicos [Hunkeler et al. 2008]. Nesta arquitetura, o serviço em nuvem atua como um Bro$k e r$, que além de gerenciar as trocas de mensagens entre os tópicos, também armazena em um banco de dados as informações de consumo publicadas pelo dispositivo inteligente instalado em cada residência. Além disso, o serviço em nuvem é responsável por executar o algoritmo de identificação de eletrodomésticos. A vantagem da utilização do protocolo MQTT está no modelo assíncrono de troca de mensagens, uma vez que a aplicação móvel não necessita realizar chamadas periódicas ao serviço de nuvem para atualizar determinada informação, visto que estas atualizações são realizadas a cada nova publicação pelos dispositivos inteligentes.

\subsection{Dispositivo Inteligente}

O dispositivo inteligente proposto neste trabalho é composto por uma Raspberry Pi conectada a um sensor de corrente ${ }^{1}$ através de um conversor analógico para digital (AD). O sensor de corrente utilizado é não invasivo, ou seja, ele não requer nenhuma mudança no circuito elétrico residencial para funcionar. A instalação do sensor é feita diretamente

\footnotetext{
${ }^{1} \mathrm{O}$ Datasheet da Raspberry $\mathrm{Pi}$ e do sensor de corrente estão disponíveis em: https://drive. google.com/drive/folders/1c9LX_pSexwqYEYl8gnwnQ-niYZ2VYFNO?usp=sharing. Acessado em: 27 de Abril de 2020.
} 
no fio de saída do quadro de registro elétrico. O sensor gera em sua saída, um sinal de corrente no domínio do tempo proporcional ao sinal de corrente demandado pelos eletrodomésticos conectados na rede elétrica. A medida máxima de corrente suportada pelo sensor é de 100 Amperes e o sinal de corrente gerado é amostrado pelo AD a uma precisão de 16 bits e a taxa de amostragem de 24000 amostras/s.

\subsection{Algoritmo de Identificação de Eletrodomésticos}

O Algoritmo 1 descreve o procedimento de coleta e processamento das informações de consumo energético. Na Linha 4 é coletado o sinal de corrente elétrica através da leitura do sensor de corrente e, no passo seguinte, o sinal coletado é utilizado para calcular o valor da potência consumida. Na Linha 6 é verificado se houve um aumento ou redução da potência de consumo, significando, respectivamente, a adição ou remoção de algum eletrodoméstico na rede elétrica residencial. Em caso afirmativo, são enviados para o serviço em nuvem as componentes harmônicas e o valor da potência consumida (Linhas 8 e 9$)$.

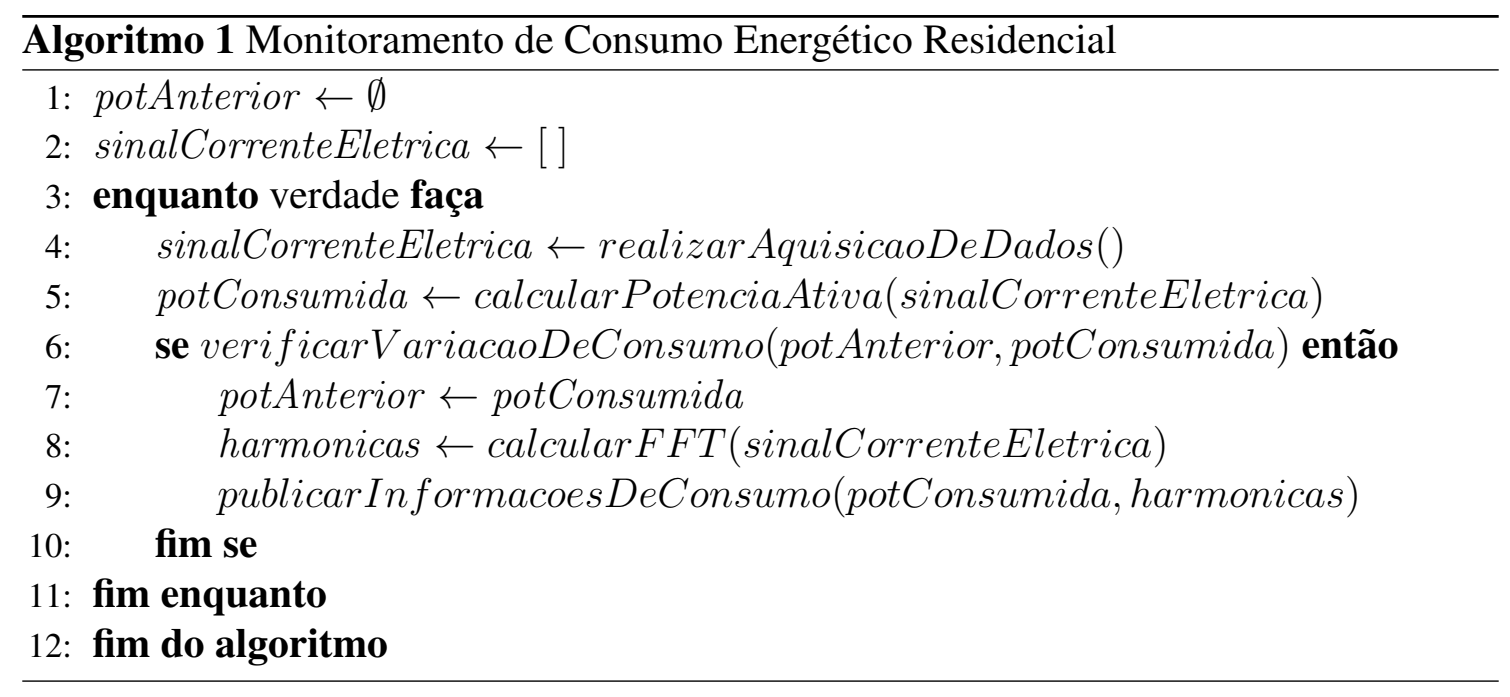

O algoritmo de identificação requer, como premissa, o acionamento e desligamento sequencial dos eletrodomésticos. Estes eventos são registrados em um banco de dados na nuvem, na qual são armazenados, também, as informações de potência consumida e as componentes harmônicas do sinal de corrente. Desta forma, é criada uma linha do tempo, que poderá ser útil para a recuperação do sistema em casos de problemas específicos, como por exemplo, a falta de energia na residência.

O Algoritmo 2 especifica os passos necessários para a identificação dos eletrodomésticos. O primeiro passo consiste em receber do dispositivo inteligente, as informações de consumo energético (Linha 4) e, em seguida, com base nas variações das amplitudes das componentes harmônicas coletadas em relação a última medição (Linha 5) é formado um conjunto de características que, por sua vez, serão classificadas por meio de um algoritmo de aprendizado supervisionado de máquina e, como resultado, é obtida a identificação do eletrodoméstico (Linha 6). Ao final (Linhas 7 a 11), é realizado uma verificação do valor de potência consumida com o objetivo de descobrir o estado (ligado ou desligado) do eletrodoméstico reconhecido. O registro destas informações são refletidas para o usuário através da aplicação móvel. 


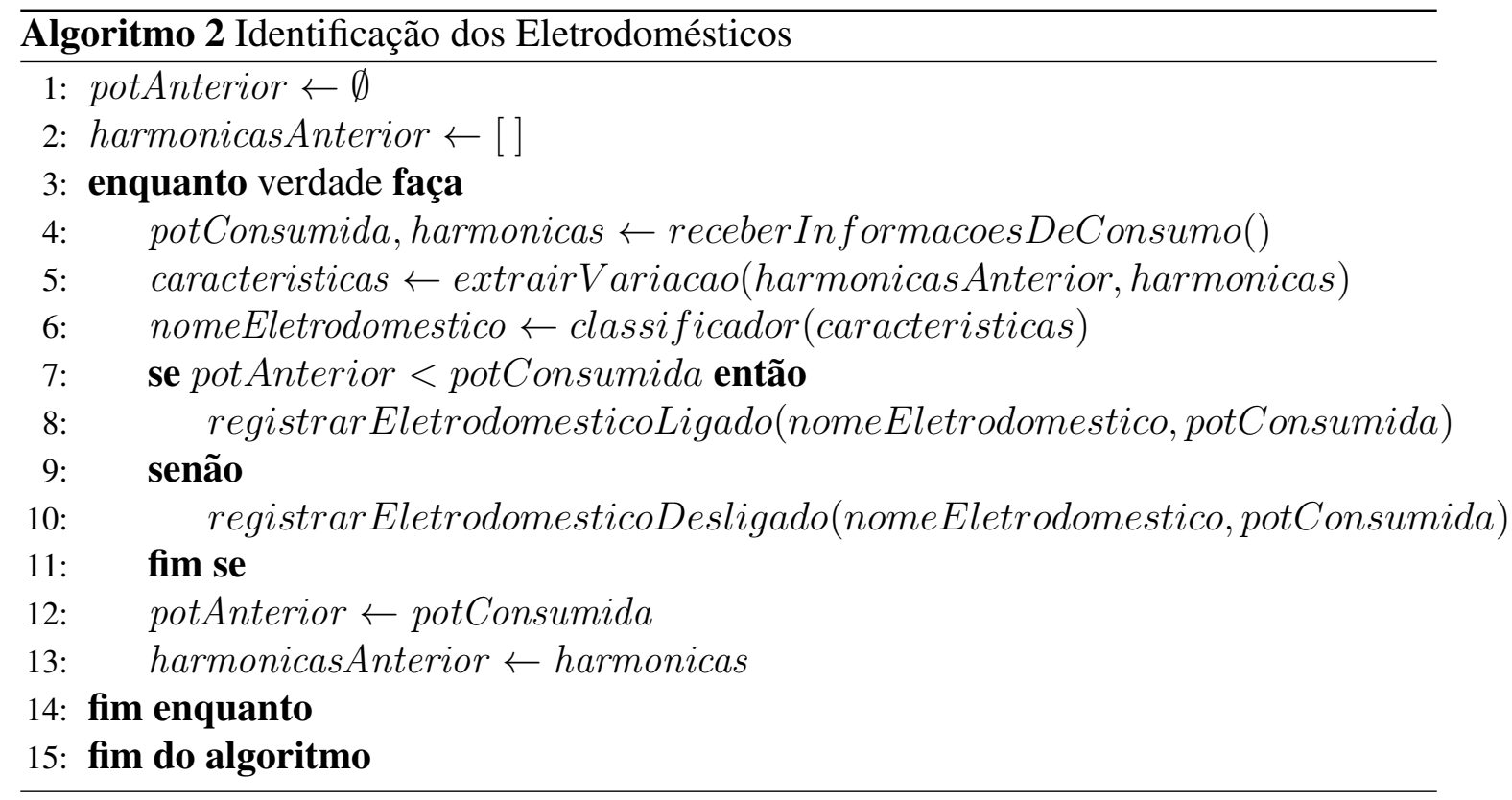

O treinamento utiliza um dataset composto pelas componentes harmônicas extraídas do sinal de consumo de diferentes tipos de eletrodomésticos. A escolha foi ocasionada devido ao fato de que cada tipo de eletrodoméstico possui uma organização exclusiva destas componentes no espectro de frequência [Wu et al. 2018]. Cada registro no dataset representa o funcionamento individual de um eletrodoméstico na rede elétrica a fim diminuir a complexidade durante a análise de sinais de consumo pelo algoritmo.

\section{Resultados e Discussão}

Nesta seção são apresentados os resultados obtidos com a solução proposta neste artigo. Foram realizados dois tipos de experimentos: o primeiro consiste em avaliar a classificação dos eletrodomésticos através de sinal de consumo individual e o segundo avalia o classificador com base no consumo agregado por vários eletrodomésticos.

\subsection{Experimento 1 - Avaliação dos Classificadores}

Para a realização deste experimento foram implementados, com o auxílio da biblioteca Scikit-learn 2, 4 diferentes tipos de algoritmos de aprendizagem supervisionada de máquina: K-Nearest Neighbor (KNN), Multi Layer Perceptron (MLP), Support Vector Machines (SVM) e Random Forests.

Os algoritmos escolhidos possuem sua própria metodologia de classificação, por exemplo o KNN é um algoritmo de clusterização e a classificação é feita através do cálculo de distância entre as entidades. Já o algoritmo MLP é baseado em uma rede neural e a identificação dos eletrodomésticos é feita por meio de um sistema de pesos existentes nas ligações entre os "neurônios", que vão se ajustando à medida que o algoritmo vai errando os resultados. Os algoritmos SVM e o Random Forests trabalham com hiperplanos e árvores de decisão, respectivamente, com o objetivo de separar as entidades do sistema com base em um conjunto de características.

\footnotetext{
${ }^{2}$ Biblioteca Scikit-learn, disponível em: https: //scikit-learn.org. Acessado em: 27 de Abril de 2020 .
} 
É importante ressaltar que não se pretende neste trabalho realizar uma otimização dos parâmetros dos algoritmos escolhidos, em vez disso foram adotados os parâmetros padrões de cada algoritmo disponibilizados pela biblioteca Scikit-learn. Em termos de diferenciação de algorítmos, o KNN utiliza o parâmetro $\mathrm{K}=5$ para a seleção dos vizinhos mais próximos, o MPL utiliza 2 camadas ocultas com 100 neurônios em cada camada, utilizando a função logística para ativação dos neurônios, o SVM utiliza o Kernel RBF para mapear as instâncias e o Random Forests possui o total de 100 árvores na floresta de decisão.

Um dataset de características foi criada a partir de uma rotina de acionamento, de maneira isolada, de um grupo de eletrodomésticos ${ }^{3}$ na rede elétrica. Cada eletrodoméstico ficou conectado por cerca de 5 segundos e o dataset foi preenchido com as harmônicas geradas pela operação deste eletrodoméstico. A Figura 2 apresenta a acurácia dos algoritmos de aprendizado de máquina na tarefa de identificação de eletrodomésticos através do dataset criado. Para a aprendizagem dos algoritmos foram considerados $70 \%$ dos registros do dataset e os demais $30 \%$ foram utilizados nos testes de classificação. $\mathrm{O}$ alto índice de acertos na identificação dos eletrodomésticos indicam que as harmônicas do sinal de corrente realmente refletem as características únicas dos eletrodomésticos analisados.

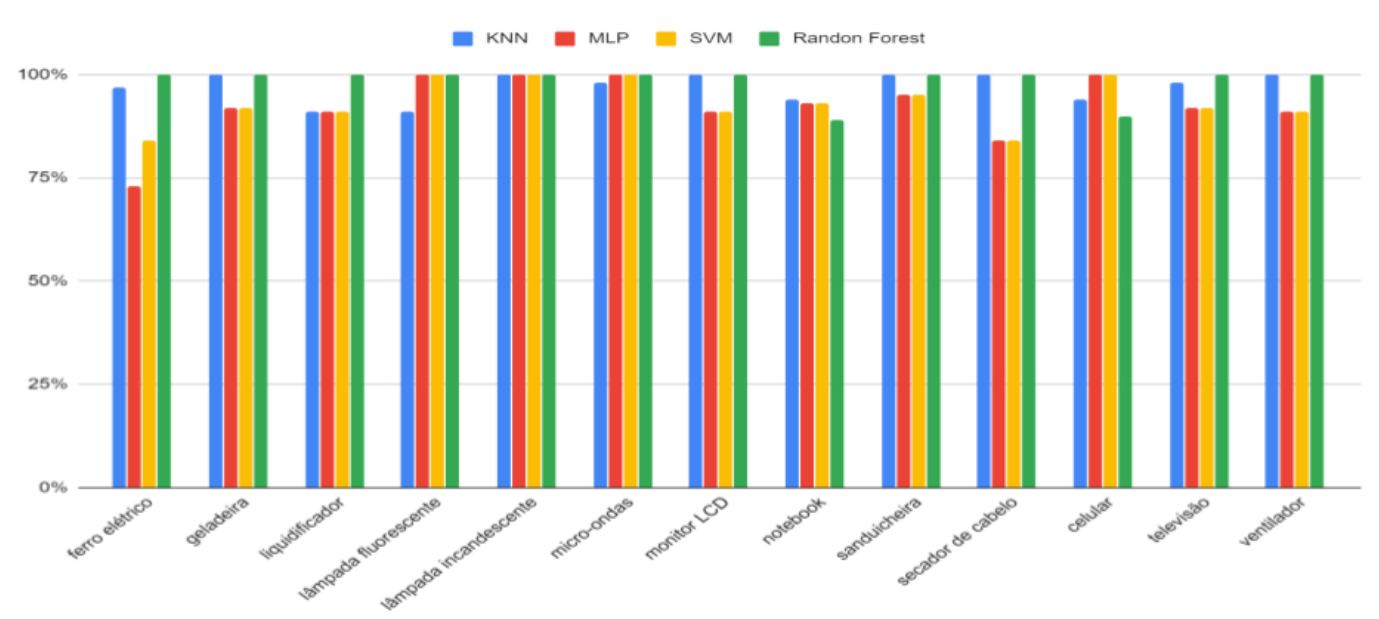

Figura 2. Acurácia dos Algoritmos na Identificação de Eletrodomésticos

\subsection{Experimento 2 - Avaliação da Solução Proposta}

Neste experimento foram criados 5 cenários de teste, cada cenário possui um conjunto de eletrodomésticos (Tabela 1). Os eletrodomésticos foram acionados sequencialmente na rede elétrica residencial, em um intervalo de tempo de 5 segundos. O algoritmo de classificação utilizado foi o KNN por ser computacionalmente menos intensivo e a pela sua boa taxa de acurácia no experimento anterior. Para cada cenário foram realizadas permutações na ordem de acionamento dos eletrodomésticos. A cada adição de um eletrodoméstico foi realizado o registro da resposta obtida pelo algoritmo de classificação. A partir dos resultados obtidos, percebe-se uma acurácia média de 97\% para todos os cenários.

${ }^{3} \mathrm{O}$ Dataset e a lista de eletrodomésticos utilizados estão disponíveis em https:// drive.google. com/drive/folders/1c9LX_pSexwqYEYl8gnwnQ-niYZ2VyFNO?usp=sharing. Acessado em: 27 de Abril de 2020. 
Tabela 1. Identificação de Eletrodomésticos com base em Sinais Compostos

\begin{tabular}{llll}
\hline Cenário & Eletrodomésticos & Acurácia & Desvio Padrão \\
\hline C1 & $\begin{array}{l}\text { Ferro de passar roupa, liquidificador e } \\
\text { geladeira }\end{array}$ & $98 \%$ & 0.0060 \\
\hline C2 & $\begin{array}{l}\text { Micro-ondas, sanduicheira, televisão e } \\
\text { secador de cabelo }\end{array}$ & $97 \%$ & 0.0070 \\
\hline C3 & $\begin{array}{l}\text { Ferro de passar roupa, sanduicheira e } \\
\text { secador de cabelo }\end{array}$ & $97 \%$ & 0.0171 \\
\hline C4 & $\begin{array}{l}\text { Televisão, liquidificador, micro-ondas } \\
\text { e notebook }\end{array}$ & $97 \%$ & 0.0033 \\
\hline C5 & Micro-ondas, ventilador e celular & $98 \%$ & 0.0048 \\
\hline
\end{tabular}

\section{Conclusão e Trabalhos Futuros}

Por um lado, observa-se que a falta de um monitoramento energético residencial acaba auxiliando no aumento do desperdício de energia elétrica. Por outro lado, com o avanço das pesquisas no tema Internet das Coisas, surge a possibilidade de utilizar sensores para coletar informações de consumo energético e protocolos para transmitir esses dados para a nuvem. Neste contexto, este artigo apresenta um sistema inteligente de monitoramento de consumo elétrico residencial que usa os conceitos de IoT com um algoritmo de identificação dos eletrodomésticos existentes em uma residência através da análise das componentes harmônicas presentes no sinal de corrente elétrica.

Como trabalhos futuros, é importante uma parametrização dos algoritmos de classificação no intuito de otimizar o desempenho dos mesmos. Além disso, também é essencial a utilização de métricas mais robustas, como a F1-Score, Recall e Matthews Correlation Coefficient para uma melhor avaliação do algoritmo de identificação de eletrodomésticos.

\section{Referências}

[Abesco 2017] Abesco (2017). Desperdício de energia atinge $\mathrm{R} \$ 61,7$ bi em três anos Abesco - Eficiência Energética.

[Andrade et al. 2017] Andrade, R. M., Carvalho, R. M., de Araújo, I. L., Oliveira, K. M., and Maia, M. E. (2017). What changes from ubiquitous computing to internet of things in interaction evaluation? In Lecture Notes in Computer Science (including subseries Lecture Notes in Artificial Intelligence and Lecture Notes in Bioinformatics), volume 10291 LNCS, pages 3-21.

[Atzori et al. 2010] Atzori, L., Iera, A., and Morabito, G. (2010). The internet of things: A survey. Computer Networks, 54(15):2787-2805.

[Azevedo et al. 2019] Azevedo, G. T., Romão, E. C., and Menegatti, C. R. (2019). Correção de distorções harmônicas em sistemas elétricos através de interferência destrutiva. $R e$ vista Brasileira de Ensino de Física, 41(3).

[Bandyopadhyay et al. 2011] Bandyopadhyay, S., Sengupta, M., Maiti, S., and Dutta, S. (2011). Role of middleware for internet of things: A study. International Journal of Computer Science and Engineering Survey, 2(3):94-105. 
[Gaikwad et al. 2015] Gaikwad, P. P., Gabhane, J. P., and Golait, S. S. (2015). A survey based on Smart Homes system using Internet-of-Things. In 4th IEEE Sponsored International Conference on Computation of Power, Energy, Information and Communication, ICCPEIC 2015, pages 330-335. IEEE.

[Gubbi et al. 2013] Gubbi, J., Buyya, R., Marusic, S., and Palaniswami, M. (2013). Internet of Things (IoT): A vision, architectural elements, and future directions. Future Generation Computer Systems, 29(7):1645-1660.

[Hunkeler et al. 2008] Hunkeler, U., Truong, H. L., and Stanford-Clark, A. (2008). MQTT$\mathrm{S}$ - A publish/subscribe protocol for wireless sensor networks. In 3rd IEEE/CreateNet International Conference on Communication System Software and Middleware, COMSWARE, pages 791-798. IEEE.

[Jacobs e Maun 2019] Jacobs, G. and Maun, J. C. (2019). Identifying washing machine consumption in supervised global electric consumption. In 2019 IEEE Milan PowerTech, PowerTech 2019. Institute of Electrical and Electronics Engineers Inc.

[Ma et al. 2017] Ma, M., Lin, W., Zhang, J., Wang, P., Zhou, Y., and Liang, X. (2017). Discover the fingerprint of electrical appliance: Online appliance behavior learning and detection in smart homes. In Proc. Internet of People and Smart City Innovation (SmartWorld/SCALCOM/UIC/ATC/CBDCom/IOP/SCI) 2017 IEEE SmartWorld, Ubiquitous Intelligence Computing, Advanced Trusted Computed, Scalable Computing Communications, Cloud Big Data Computing, pages 1-8.

[Santos et al. 2016] Santos, B. P., Silva, L., Celes, C., Borges, J. B., Neto, B. S. P., Vieira, M. A. M., Vieira, L. F. M., Goussevskaia, O. N., and Loureiro, A. (2016). Internet das coisas: da teoria à prática. Minicursos SBRC-Simpósio Brasileiro de Redes de Computadores e Sistemas Distribuıdos.

[Semwal et al. 2014] Semwal, S., Shah, G., and Prasad, R. S. (2014). Identification residential appliance using NIALM. In 2014 IEEE International Conference on Power Electronics, Drives and Energy Systems, PEDES 2014, pages 1-6. IEEE.

[Temneanu 2014] Temneanu, M. (2014). Appliance characterization based on spectral components analysis. In 2014 International Conference and Exposition on Electrical and Power Engineering (EPE), pages 710-714. IEEE.

[Wu et al. 2018] Wu, X., Han, X., Liu, L., and Qi, B. (2018). A load identification algorithm of frequency domain filtering under current underdetermined separation. IEEE Access, 6:37094-37107.

[Yokotani e Sasaki 2017] Yokotani, T. and Sasaki, Y. (2017). Comparison with HTTP and MQTT on required network resources for IoT. In ICCEREC 2016 - International Conference on Control, Electronics, Renewable Energy, and Communications 2016, Conference Proceedings, pages 1-6. IEEE.

[Zhuang et al. 2019] Zhuang, M., Shahidehpour, M., and Li, Z. (2019). An Overview of Non-Intrusive Load Monitoring: Approaches, Business Applications, and Challenges. In 2018 International Conference on Power System Technology, POWERCON 2018 Proceedings, pages 4291-4299. Institute of Electrical and Electronics Engineers Inc. 